C-A/AP/\#198 April 2005

\title{
Observations related to Beam Transition Pressure Rise
}

\author{
S.Y. Zhang
}

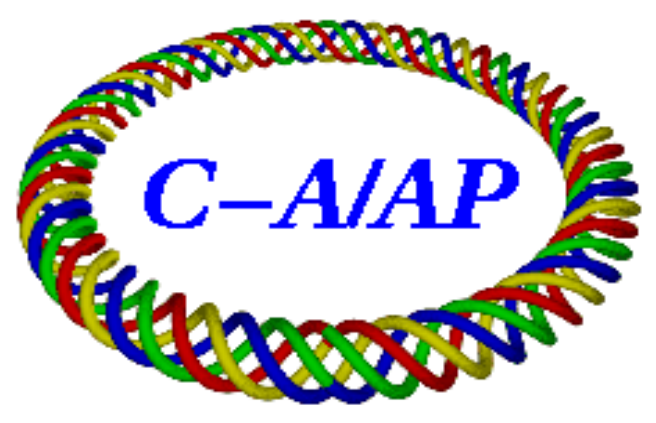

Collider-Accelerator Department Brookhaven National Laboratory Upton, NY 11973 


\title{
Observations related to Beam Transition Pressure Rise
}

\author{
S.Y. Zhang
}

\section{Introduction}

Two observations related to beam transition pressure rise, mainly in 2005 copper run, are reported in this note.

One is that the transition pressure rise at interaction regions IR10, IR12, and IR2 in the $\mathrm{Cu}-\mathrm{Cu}$ run have shown clear difference from the d-Au run and the $\mathrm{Au}-\mathrm{Au}$ run. This difference can be reduced if a $\mathrm{Z}$ factor of the beam ions is phased in. In specific, with larger $Z$ the slope of the transition pressure rise with respect to the total beam intensity is larger. If confirmed, then it may have some impact on the heavier ions' program at RHIC, such as Uranium.

The second one is that halo scraping at the beam transition has been observed at the anti-grazing ridges installed 40 meters away from IP6. Neither dynamic aperture nor momentum aperture can explain this scraping. A possible beam momentum spread associated non-linear effect at the triplet is suspected for producing such halos, reaching larger than 8 times of $\mathrm{rms}$ beam size. If confirmed, this may help to explain the transition pressure rise, and the RHIC warm section electron cloud as well.

\section{$2 \quad \mathrm{Z}$ dependence in transition pressure rise}

In Figure 1, the transition pressure rise of IR10, IR12, and IR2 in d-Au run 2003, $\mathrm{Au}-\mathrm{Au}$ run 2004, and $\mathrm{Cu}-\mathrm{Cu}$ run 2005 are shown against the total beam intensity in a charge unit of $10^{9}$ fully stripped gold ions.

Most high intensity ramps in $\mathrm{d}-\mathrm{Au}, \mathrm{Au}-\mathrm{Au}$, and $\mathrm{Cu}-\mathrm{Cu}$ operations, including low energy runs for $\mathrm{Au}-\mathrm{Au}$ and $\mathrm{Cu}-\mathrm{Cu}$, are included in Figure 1. About 14 beam study ramps in 2005 with typical $4.5 \times 10^{9} \mathrm{Cu}$ ions $\left(1.65 \times 10^{9}\right.$ 
Au ions charge equivalent) per bunch, in 112 bunch mode, are not included. The transition pressure rise of these study ramps is clearly electron cloud dominant, which reaches $10^{-8}$ Torr or higher, but the total beam intensity is at some $70 \times 10^{9} \mathrm{Au}$ ions equivalent (only about 40 bunches injected). These study ramps with high bunch intensity and shortest bunch spacing in RHIC, $108 \mathrm{~ns}$, were intended for future development.

There is clear dependence of the pressure rise slope vs. beam intensity on the average $\mathrm{Z}$ of the beam, 79 for $\mathrm{Au}-\mathrm{Au}, 40$ for $\mathrm{d}-\mathrm{Au}$, and 29 for $\mathrm{Cu}-$ $\mathrm{Cu}$. Note that previously no clear difference of deuteron and gold beam ions on the transition pressure rise was identified based on d-Au run data [1], perhaps the gap between the higher gold and high deuteron beams is not large enough to identify this effect. Also, in Au-Au run 2004, the steeper slope of the transition pressure rise against the beam intensity was noticed, but only after $\mathrm{Cu}-\mathrm{Cu}$ run 2005, it can be identified due to $\mathrm{Z}$ dependence.

Most d-Au ramps at lower intensities are with 56 bunch mode, and others are with 112 bunch mode. Most $\mathrm{Au}-\mathrm{Au}$ ramps are with 45 bunch mode, others are with 56, 61, and 68 bunch modes. Typical and most of $\mathrm{Cu}-\mathrm{Cu}$ ramps are with 37 bunch mode, rest are with 28, 40, 42, 45, 48, and 56 bunch modes.

No bunch mode, and hence bunch spacing, dependence of the transition pressure rise can be identified at all IRs in d-Au run [1]. For Au-Au run, no dependence was shown at IR10 and IR2, but some dependence was shown at IR12 [2]. In $\mathrm{Cu}-\mathrm{Cu}$ run, there is some dependence at all 3 IRs, shown in Figure 2. This dependence indicates some contributions of the electron multipacting in the transition pressure rise, nevertheless, electron cloud seems not a dominant factor. It is interesting to note from Figure 2 that the slopes of the pressure rise with different bunch modes are not changed, indicating that the electron multipacting just contributes some additional pressure rise.

Vacuum gauge at IR12 (g12.pwx) reported elevated static pressure to about $3 \times 10^{-10}$ Torr in Au-Au run, shown in Figure 1 . Some leaks in the gauge were found, but the data shown in Figure 1 look usable.

The transition pressure rise of IR2 in $\mathrm{Cu}-\mathrm{Cu}$ run had large uncertainties with respect to beam intensities. For ramps with same intensities and just hours apart, pressure rise may differ by more than 2 orders of magnitude. This is not understood.

Three ramps in $\mathrm{Cu}-\mathrm{Cu}$ run have higher pressure rise with respect to the beam intensity, most noticeably in IR2, and also identifiable in IR10 and IR12. These are the ramp 5849 with total intensity of $105.3 \times 10^{9} \mathrm{Au}$ ions 
equivalent in 48 bunch mode, 5851 with $109 \times 10^{9}$ in 56 bunch mode, and 5852 with $120.4 \times 10^{9}$ in 48 bunch mode. These 3 ramps took place in early run, within 3 hours.

We have some discussions.

1. In general, the slope of the transition pressure rise vs. beam intensity is different for Au-Au with $Z=79$, d-Au with average $Z=40$, and $\mathrm{Cu}-\mathrm{Cu}$ with $Z=29$. The largest slope with the $\mathrm{Au}-\mathrm{Au}$ beams can be clearly identified from $\mathrm{d}-\mathrm{Au}$ and $\mathrm{Cu}-\mathrm{Cu}$ runs. A rough fitting of $Z^{-k}$ with $k=1 \sim 2$ can unify the slopes in different degrees at these IRs. With this observation, one may predict that the heavier beam ions will imply higher transition pressure rise . For example, Uranium with $Z=92$ may have steeper slope of transition pressure rise vs. beam intensity than the gold ions.

2. The transition pressure rise had directly caused the experimental background problem in d-Au run. High transition pressure rise needed up to 10 hours to reduce to acceptable level. In Au-Au run, it was mainly the beam rebucketing pressure rise that caused the experimental background problem. However, at high beam intensity, $>100 \times 10^{9} \mathrm{Au}$ ions, the transition pressure rise seems also relevant. In $\mathrm{Cu}-\mathrm{Cu}$ run, the pressure rise induced background is not a limit of the beam intensity, but the margin is not large.

3. It is interesting to note that the typical total beam intensity is $95 \times 10^{9}$ $\mathrm{Au}$ ions for $\mathrm{Au}-\mathrm{Au}$ run, it is $110 \times 10^{9} \mathrm{Au}$ ions equivalent for $\mathrm{d}-\mathrm{Au} \mathrm{run}$, and $125 \times 10^{9} \mathrm{Au}$ ions equivalent for $\mathrm{Cu}-\mathrm{Cu}$ run. In the case of IR10, transition pressure rises with these intensities are all at about $10^{-8}$ Torr. This is probably not just a coincidence, but rather an indicator of the machine operation limit.

4. One possible mechanism of the $\mathrm{Z}$ dependence of the transition pressure rise on the beam species is the beam-gas ionization. The beam gas ionization generated ions are pushed to wall by beam, but the ions' impact energy is too low to produce meaningful neutral particles [3]. On the other hand, these ions may help the secondary electrons to stay longer, and hence to cause electron multipacting. As discussed, nontrivial electron multipacting is believed to take place at some locations and sometimes in the transition pressure rise. Nevertheless, electron 
cloud seems not dominant in the operation ramps, for the reasons shown below.

- One reason is that at the beam rebucketing the beam peak current is often higher than at the transition, yet the pressure rise at the rebucketing is less pronounced and sometimes missing. A good example is that at IR2, pressure rise was absent at the beam rebucketing in almost entire $\mathrm{Au}-\mathrm{Au}$ run 2004, in contrast with the transition pressure rise shown in Figure 1. Both beam peak current and beam potential at the rebucketing are higher than that at the transition, which are supposed to be more favorable in electron multipacting. As an example, at SPS it is observed that the electron cloud is stronger at higher energies in accelerations [4].

- The second reason is that the threshold of the transition pressure rise is about $85 \times 10^{9} \mathrm{Au}$ ions equivalent for 28 bunches to 112 bunches, i.e., bunch spacing from $432 \mathrm{~ns}$ to $108 \mathrm{~ns}$. If the transition pressure rise is mainly caused by the electron cloud, then this implies that the electron cloud threshold of bunch intensity against bunch spacing is linear. This is not agreeable with the machine observation and code simulations. Again at SPS, it is observed that the electron cloud threshold at dipole is $0.3 \times 10^{11}$, $0.6 \times 10^{11}$, and $1.2 \times 10^{11}$ protons per bunch for the bunch spacing of $25 \mathrm{~ns}, 50 \mathrm{~ns}$, and $75 \mathrm{~ns}$ [5], the relation is not linear. This relation is agreeable with the observations in many other machines, and it can also be explained by simulations [6].

- No meaningful electron multipacting signal has been detected by the electron detectors at the beam transition for all ramps shown in Figure 1. Electron signals at transition were detected, nevertheless, in the high bunch intensity 112 bunch mode beam study ramps in $\mathrm{Cu}-\mathrm{Cu}$ run, which are clearly electron multipacting dominant, as mentioned above.

5. Another possible mechanism of the $\mathrm{Z}$ dependence in transition pressure rise is the interaction of the beam ion and the chamber wall. Usual beam losses are believed irrelevant in the transition pressure rise [7], explained by the not-shallow-enough incident angles in these 
beam losses. The beam halo scraping with very shallow incident angle, however, could imply large ion desorptions, and hence to be responsible for the transition pressure rise. This is the subject of the second observation.

\section{Halo scraping at the beam transition}

Prior to the $\mathrm{Cu}-\mathrm{Cu}$ run, anti-grazing ridges [8] have been installed at the Q3-Q4 straight sections, Bi5 for blue and Yo5 for yellow. The ridges are installed for beam study of the effect on electron cloud threshold. In Figure 3 , the chamber size, the beam size at the transition, and the location of the ridges are illustrated in the region from the interaction point IP to 95 meters beyond.

The beam loss monitor y5- $\operatorname{lm} 3.2$ is located at 3,794 $\mathrm{m}$ in the ring, which happens to be at the ridges that are $39.4 \mathrm{~m}$ away from IP6, indicated in Figure 3 as Ridge 1. The loss monitor is placed between the Yellow and Blue pipes, sensing losses of both beams. During the entire $\mathrm{Cu}-\mathrm{Cu}$ run, persistent beam transition loss has been observed at this loss monitor. The loss is not large, below a few hundred counts, but it is observable for all ramps.

Some discussions are as follows.

1. Usual beam normalized emittance around the transition is about $\epsilon_{N, 95 \%}=$ $10 \pi \mu m$, here we take it as $\epsilon_{N, 95 \%}=20 \pi \mu m$. At the transition, with $\beta^{*}=5 \mathrm{~m}$ at IP6, the Yellow beta function at the location of Ridge 1 is $\beta_{v}=250 \mathrm{~m}$ and $\beta_{h}=100 \mathrm{~m}$, the half beam size is $a_{v}=1.47 \mathrm{~cm}$ and $a_{h}=0.93 \mathrm{~cm}$, as shown in Figure 3 . The corresponding $\mathrm{rms}$ beam size is $\sigma_{v}=0.60 \mathrm{~cm}$ and $\sigma_{h}=0.38 \mathrm{~cm}$. With the height of the ridge, $0.7 \mathrm{~cm}$, and the radius of the chamber, $6 \mathrm{~cm}$, the aperture there is 5.3 $\mathrm{cm}$. The vertical separation bump at the IP6 is usually $\pm 5 \mathrm{~mm}$, which extends to the location of Ridge 1 . With this offset, therefore, Ridge 1 poses an aperture limit at $8 \sigma_{v}$. With the beam momentum spread of $d p / p= \pm 0.42 \%$ at transition, the dispersion function $D_{h}=0.14 \mathrm{~m}$ introduces less than $1 \mathrm{~mm}$ size enlargement. Ridge 1 therefore poses a horizontal limit at $15 \sigma_{h}$. The current understanding of either dynamic aperture and/or momentum aperture cannot explain the transition loss at this location.

2. The fact of the typical small loss and the lost particles reaching such 
large aperture indicates that this is the halo scraping. The halo measurement of less than $10^{-4}$ of the peak signal at $>8 \sigma$ of the beam has been reported at PSR [9], where the space charge and foil scattering are suspected for the halo formation. For halo scraping at the transition in RHIC, the loss looks like associated with the beam momentum spread. In the two examples below, the halo scraping induced beam loss is shown, together with the model beam momentum spread.

- In Figure 4, this beam loss is shown for a normal operation, ramp 6196. The bunch intensity was $4.3 \times 10^{9} \mathrm{Cu}$ ions, and the fill pattern was 37 bunches. The RF voltage at the transition was a normal $300 \mathrm{kV}$. The beam loss is started 50 seconds after the ramping, 20 seconds before the transition.

- In Figure 5, the beam loss for ramp 6005 is shown. This is a beam study ramp, 24 bunches in 112 bunch mode are ramped in Yellow ring, with the bunch intensity of $5 \times 10^{9} \mathrm{Cu}$ ions. The RF voltage is reduced from $300 \mathrm{kV}$ to $100 \mathrm{kV}$ around the transition, which affected the beam momentum spread. The RF voltage and the momentum spread are also shown. The beam loss is not closely coincided with the model momentum spread, however, it did show some wiggles.

3. The mechanism of the halo formation at the RHIC beam transition is not yet understood. Several possible factors are listed below for discussion.

- The beam peak current is high at the transition, typically 5 amps. The high beam current alone cannot produce halos. Also, the peak current at the beam rebucketing is comparable with, and often higher than, the one at the transition, yet the loss at Ridge 1 is less persistent at the beam rebucketing, sometimes it is absent.

- There is also large tune spread at the transition from chromatic and octupolar effects. However, it is not clear how the tune spread to drive some beam particles to this large aperture.

- Beam instabilities have potential to produce halos. Usual beam instabilities are observed above transition, which is not coincided with the transition loss with the halo scraping started way ahead of the transition. 
- The beam momentum spread reaches $d p / p= \pm 0.42 \%$ at the transition, it is reduced to less than $0.1 \%$ in the subsequent energy acceleration. At the beam rebucketing, when the bunches are captured by the $200 \mathrm{MHz}$ storage cavities, the beam momentum spread jumped to $0.31 \%$ with the typical storage RF voltage of $3.5 M V$ in the copper run. The beam momentum spread at the rebucketing is lower than the one at the transition. This may indicate that the beam momentum spread is a more relevant factor compared with the peak current. In Figure 6, this is illustrated.

4. Several ramps with blue beam have smaller losses, therefore, it is suspected that yellow beam might contribute more than blue in $\mathrm{Cu}-\mathrm{Cu}$ run at the location of Ridge 1 . Immediately upstream of Ridge 1 of yellow beam, it is the Q1-Q3 triplet. One possibility is that the beam momentum spread related non-linear effect at the triplet may pump out some beam halos, reaching a large aperture like at Ridge 1 at the downstream locations.

Finally, we discuss the effect of the transition halo scraping.

Most crucial factor in terms of ion desorption probably is the incident angle. Beam study has shown that with the incident angle of $1 \mathrm{mrad}$, the gold ion desorption rate at the RHIC injection energy is about $2 \times 10^{4}[1,10]$. With a limited beam loss at the transition, this desorption rate cannot explain the transition pressure rise.

It is also noticed that the beam particles in the core, with betatron oscillations, will not have incident angles much smaller than 1 mrad once reaching chamber wall. This is agreeable with the fact that most beam loss did not create much pressure rise.

In fact, considering the relevant surface with the thickness of $10 \mathrm{~nm}$ to $100 \mathrm{~nm}$, from where the secondary particle might escape, the energy dumped there by the beam ions with the incident angle of $1 \mathrm{mrad}$ is limited. To have very large ion desorption, such as that observed at RHIC [10], the incident angle must be much smaller than $1 \mathrm{mrad}$. This is not achievable with the normal beam, but might be achievable with the halo type of particles.

The existing understanding of halos under non-linear resonances may form small islands in phase space, and the particles confined there could be performing very shallow scraping at the straight sections. Given the ample energy available for the beams at RHIC, a part of the beam halo particles 
could release large amount of neutral particles, provided that the energy is dumped on the relevant surface. The mechanism of the halo formation at the beam transition therefore is worth a detailed study.

\section{Acknowledgment}

The author would like to thank A. Fedotov, V. Ptitsyn, and S. Tepikian for helpfull discussions.

\section{References}

\section{References}

[1] S.Y. Zhang et al, 'RHIC pressure rise,' EPAC'04, Lucerne, Switzerland, July 2004.

[2] W. Fischer, 'Electron clouds and vacuum pressure rise in RHIC,' HB2004, Beinshein, Germany, Oct. 2004.

[3] S.Y. Zhang, 'RHIC run-away type pressure rise,' C-AD AP, 190, Jan. 2005.

[4] J.M. Jimenez, Pressure rise workshop, BNL, Dec. 2003.

[5] G. Arduini, Chamonix XIII, Jan. 2004.

[6] F. Zimmermann, ECLOUD'04, Panel discussion, Napa, California, Apr. 2004.

[7] S.Y. Zhang et al, 'RHIC pressure rise and electron cloud,' PAC'03, 54, Portland, Oregon, 2003.

[8] P. Thieberger et al, PRST-AB, Vol. 7, 093201, Sep. 2004.

[9] R. Macek, Halo’03, Montauk, N.Y. May 2003.

[10] S.Y. Zhang, 'Ion desorption at RHIC,' HB-2004, Beinshein, Germany, Oct. 2004. 

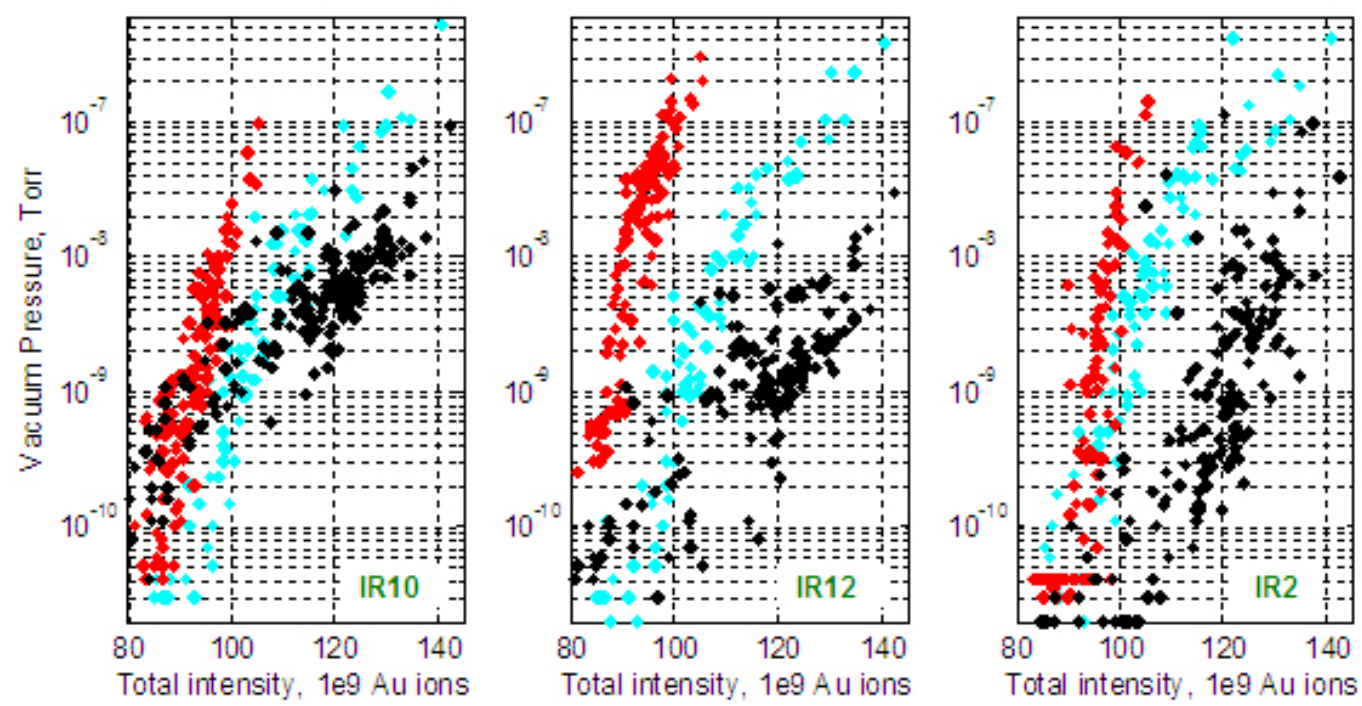

Figure 1: Transition pressure rise of interaction regions IR10, IR12, and IR2 in d-Au run 2003, $\mathrm{Au}-\mathrm{Au}$ run 2004, and $\mathrm{Cu}-\mathrm{Cu}$ run 2005, against total beam intensity in a charge unit equal to $10^{9}$ fully stripped gold ions. CYAN dots are for $\mathrm{d}-\mathrm{Au}$ run, $\mathrm{RED}$ for $\mathrm{Au}-\mathrm{Au}$ run, and BLACK for $\mathrm{Cu}-\mathrm{Cu}$ run. Most high intensity operation ramps are included. There is clear dependence of the slope of the pressure rise vs. intensity on $\mathrm{Z}$ of the beam ions, which is 79 for $\mathrm{Au}-\mathrm{Au}$, average 40 for $\mathrm{d}-\mathrm{Au}$, and 29 for $\mathrm{Cu}-\mathrm{Cu}$. 

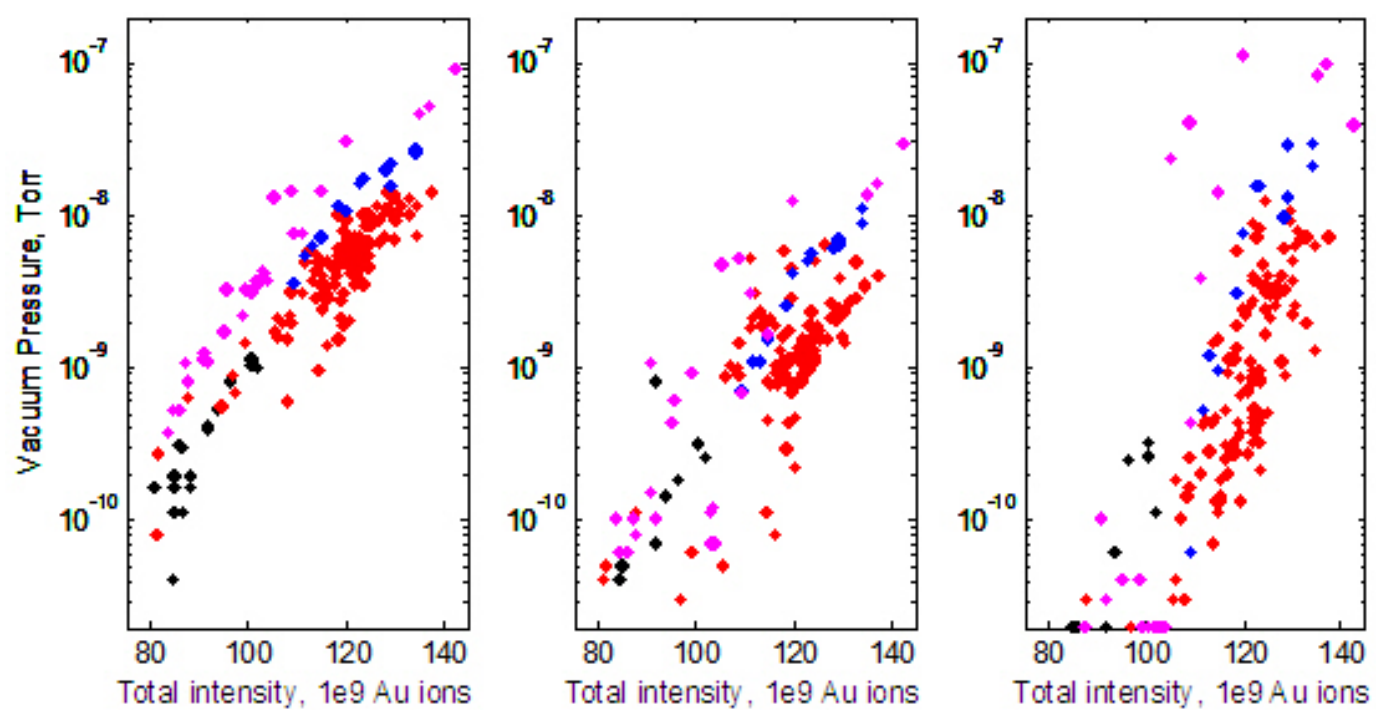

Figure 2: Bunch mode dependence of transition pressure rise in $\mathrm{Cu}-\mathrm{Cu}$ run 2005, indicating some contributions from the electron multipacting. From left to right is IR10, IR12, and IR2. Black dots are for 28 bunch mode, red is for 37 bunch mode, blue is for 40 and 42 bunch mode, and magenta is for 45, 47, 48, and 56 bunch modes. Note that the slopes of the pressure rise with different bunch modes are the same, indicating that the electron multipacting just contributes some additional pressure rise. 


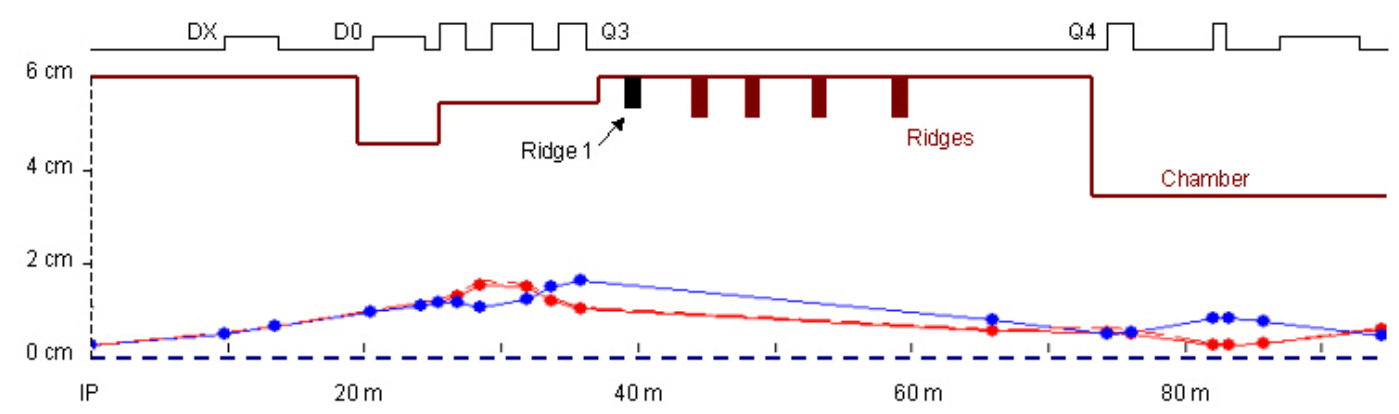

Figure 3: Illustration of chamber, ridges, and half beam size from the interaction point IP to 95 meters beyond. The half beam size is for normalized emittance of $20 \pi \mu \mathrm{m}$, with $\beta^{*}=5 \mathrm{~m}$ at the transition. Just take one beam as example, blue line is for vertical beam size, red line is for horizontal, where the dashed line shows the dispersion effect with the beam momentum spread of $d p / p= \pm 0.42 \%$ at the transition. Five anti-grazing ridges are installed in Bi5 and Yo5 each, located at $39.4 \mathrm{~m}, 44.6 \mathrm{~m}, 48 \mathrm{~m}, 53.8 \mathrm{~m}$ and $59 \mathrm{~m}$ from the IP6. Ridges have the height of $1 \mathrm{~cm}$, but the one at $39.4 \mathrm{~m}$ is $0.7 \mathrm{~cm}$. This ridge is in black color, and indicated as Ridge 1. 


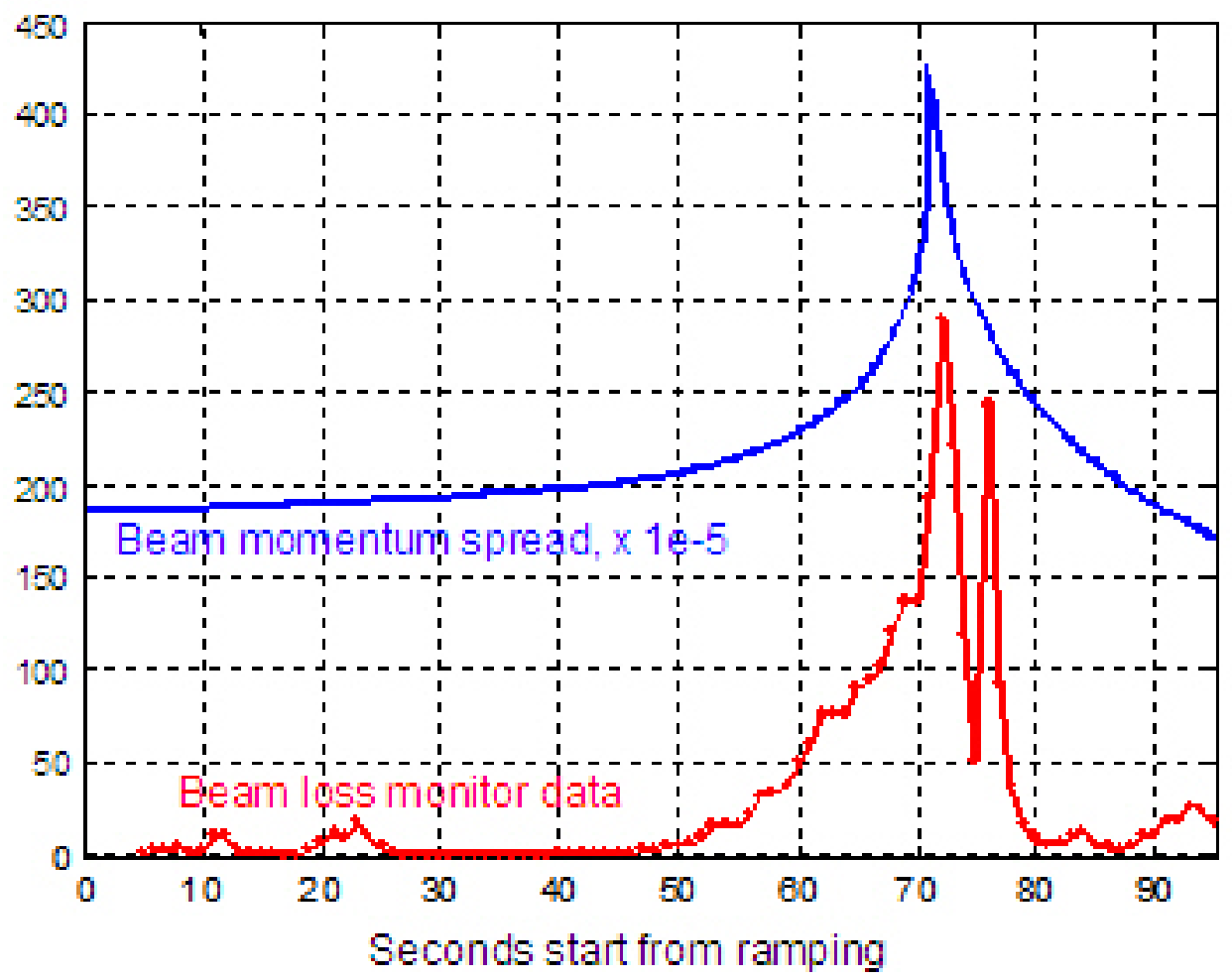

Figure 4: Beam loss at the loss monitor located at the $0.7 \mathrm{~cm}$ ridge, 39.4 $m$ away from IP6. Ramp 6196, with 37 bunch mode, $4.3 \times 10^{9} \mathrm{Cu}$ ions per bunch, in a normal operation. RF voltage is $300 \mathrm{kV}$ at the transition. The model beam momentum spread is shown for comparison. The beam loss is started 50 seconds after the ramping, 20 seconds before the transition. 


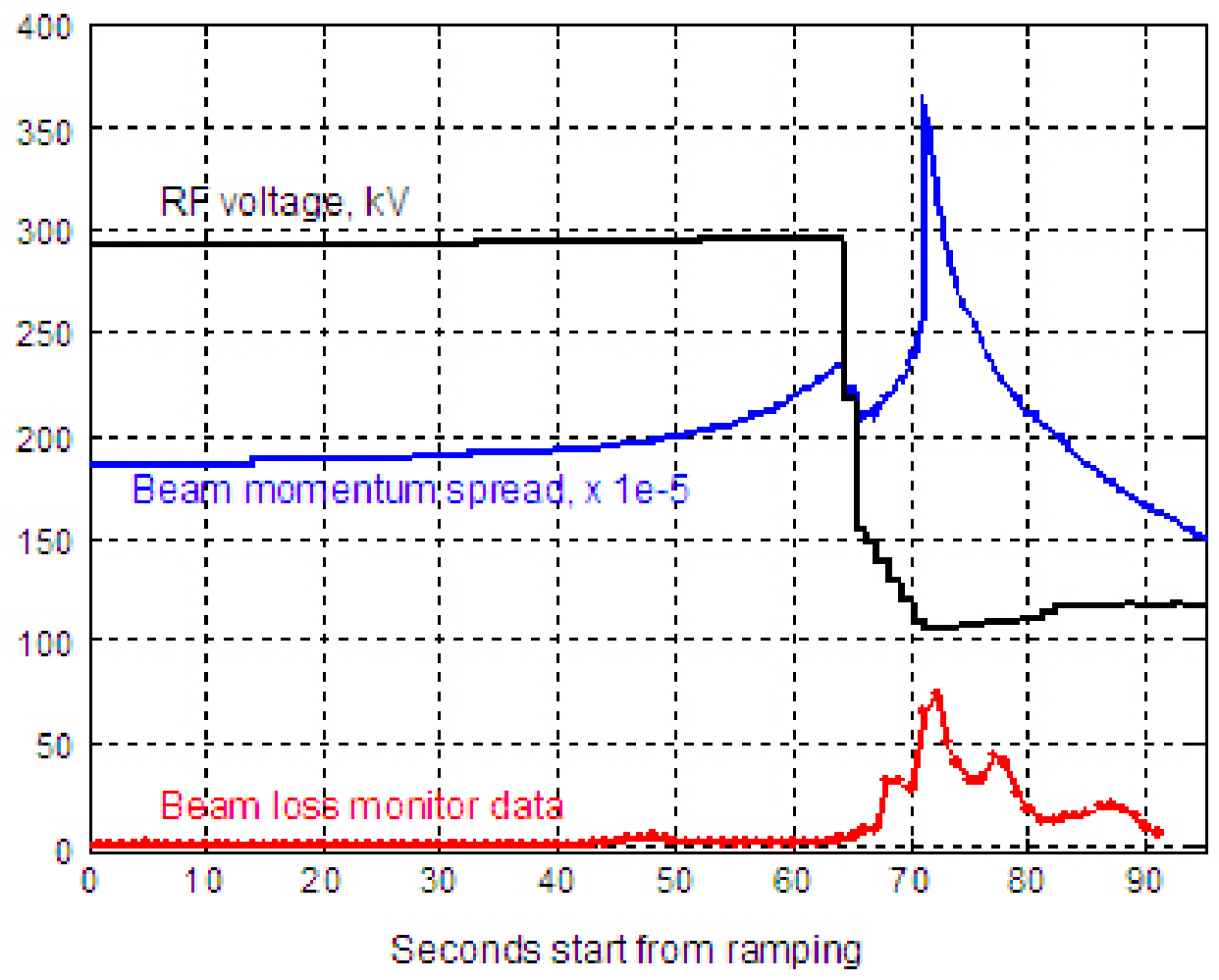

Figure 5: Beam loss at the loss monitor located at the $0.7 \mathrm{~cm}$ ridge, $39.4 \mathrm{~m}$ away from IP6. Ramp 6005 in beam study, with 24 bunches in 112 bunch mode, $5 \times 10^{9} \mathrm{Cu}$ ions per bunch injected into each ring, but the Yellow beam is suspected to contribute more in the beam loss at Ridge 1. Yellow $\mathrm{RF}$ voltage is reduced from $300 k V$ to $100 k V$ at the transition. The RF voltage and the momentum spread are also shown. The beam loss is not closely coincided with the model momentum spread, however, it did show some wiggles. 


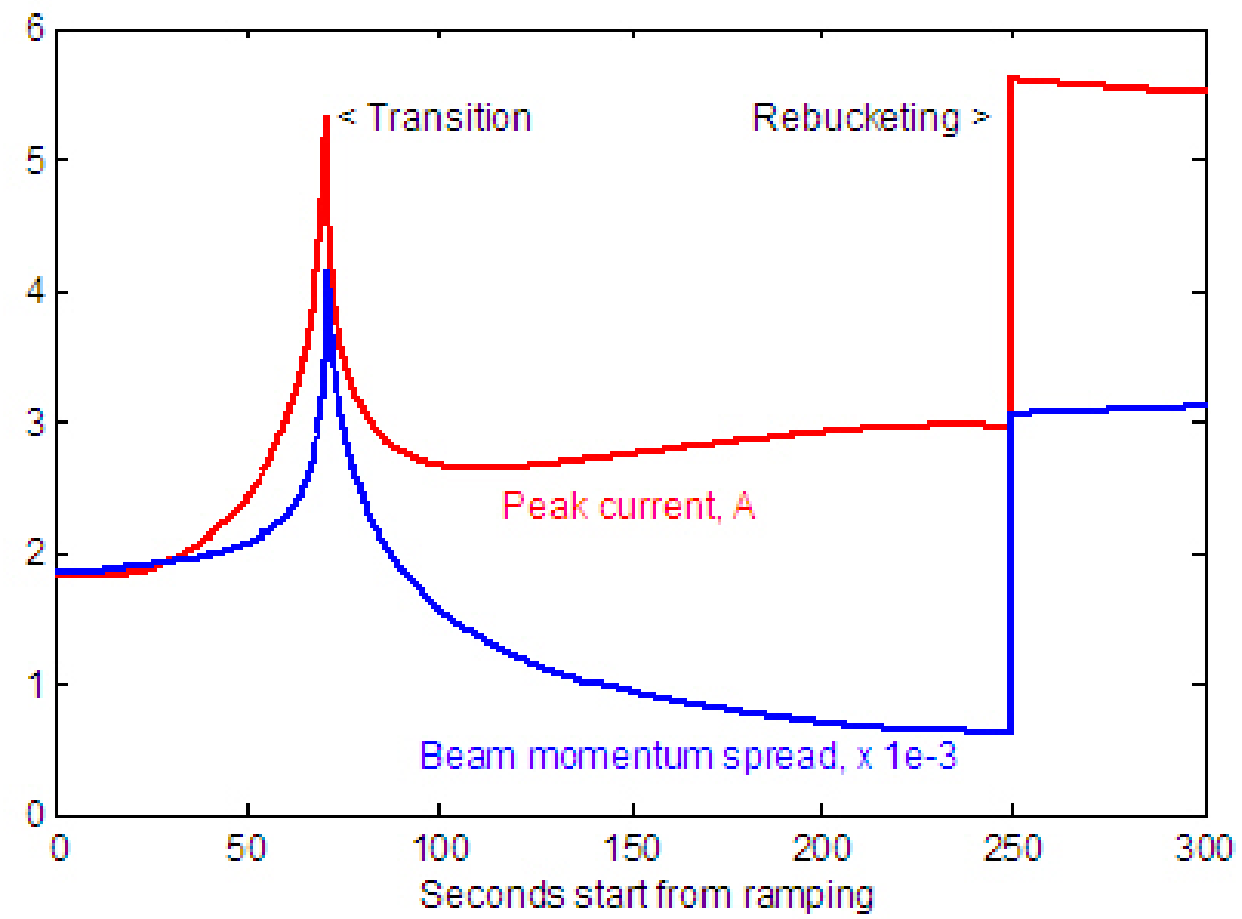

Figure 6: Illustration of beam peak current and momentum spread along the ramp in $\mathrm{Cu}-\mathrm{Cu}$ run at $\mathrm{RHIC}$. The acceleration $\mathrm{RF}$ voltage is $300 \mathrm{kV}$, and beam rebucketing voltage is $3.5 M V$. Bunch intensity is $5 \times 10^{9} \mathrm{Cu}$ ions. The bunch peak current is verifiable by the wall current monitor data, which is $5.3 A$ at the transition and $5.6 A$ at the rebucketing. The beam momentum spread is from the model, but can be verified from the bunch length and the $\mathrm{RF}$ voltage, which is $0.42 \%$ at the transition and $0.31 \%$ at the rebucketing. 